\title{
Perspective Piece Free Market Availability of Rapid Diagnostics Will Empower Communities To Control Malaria in India
}

\author{
Manju Rahi ${ }^{1}$ and Amit Sharma ${ }^{2,3^{*}}$ \\ ${ }^{1}$ Indian Council of Medical Research, New Delhi, India; ${ }^{2}$ National Institute of Malaria Research, New Delhi, India; ${ }^{3}$ Molecular Medicine, International \\ Centre for Genetic Engineering and Biotechnology, New Delhi, India
}

\begin{abstract}
Globally, malaria incidence has declined but further reductions in malaria are not evident in many countries. In addition to the public health approaches for tackling malaria, involvement of the private sector is vital because the private sector plays a central role in healthcare delivery to the masses. In India, malaria management is primarily provided through government programs, nonetheless, significant numbers of fever patients continue to seek healthcare in the private sector. The private sector in India is comprised of formal healthcare (qualified and approved), informal healthcare (unqualified, untrained), including traditional healers. Commercial channels for the procurement of quality-assured malaria diagnostics like rapid diagnostic tests via pharmacies or other approved outlets would empower Indian populations to self-detect malaria without delay. Easier access would minimize the diagnostic time gap, reduce costs to the patient, prevent inappropriate malaria treatment, and foster timely treatment of both malaria and non-malaria infections. Commercially available rapid diagnostic tests in the hands of the people could be an important tool in our fight against malaria.
\end{abstract}

Despite substantial decline in malaria incidence, India continues to suffer from a high burden of approximately 5.6 million estimated cases per year, as reported for 2019 in the 2020 World Malaria Report. ${ }^{1}$ India contributed $88 \%$ of cases and $86 \%$ of deaths to the WHO Southeast Asia Region in 2019. In the same year, 348 million rapid diagnostic tests (RDTs) were sold by manufacturers, and 267 million were distributed by national malaria programs globally. In India, approximately 134 million cases of malaria were suspected, and approximately 20 million RDTs were examined. ${ }^{1}$ Despite the aforementioned numbers, the Indian program for malaria control and elimination reported 330,000 cases and 77 deaths in 2019 based on public sector data only. ${ }^{2}$

The National Malaria Eradication Program in India (established in 1958) initially recommended presumptive treatment of all fever cases. Case detection was directed toward foci identification rather than individual case management. From 1982 to 2007, the national drug policy promoted presumptive treatment. ${ }^{3}$ Presumptive treatments are wasteful of resources, fuel the emergence of drug resistance in parasite populations, and delay the accurate diagnosis of acute and/or chronic infections. In 2010, the WHO revised their recommendations and made parasitological confirmation (through microscopy or RDTs) of infection mandatory prior to treatment with artemisinin-based combination therapy (ACT), a strategy referred to as "test-and-treat". ${ }^{4}$ Mirroring the WHO recommendations, the current Indian Drug policy (2013) now requires all suspected cases of malaria to be tested by microscopy or with RDTs, and only positive cases are administered antimalarial treatment. ${ }^{5}$ In India, the diagnosis and management of malaria is primarily conducted via the public health sector. However, it is well known that a substantial proportion of population accesses the private sector for their healthcare needs, including for febrile illnesses. Therefore, it is imperative that private caregivers like doctors, supporting workers, and drug storekeepers are included in the ambit and provided with RDTs for correct and timely diagnosis of malaria. Similarly, involvement of private sector in distribution and sale of insecticide

*Address correspondence to Amit Sharma, Sector 8, Dwarka, New Delhi 110 077, India. E-mail: directornimr@gmail.com impregnated bednets has also been proposed. ${ }^{6}$ Since their advent in the early 1990s, RDTs have circumvented the challenges posed by microscopy in the field, and especially so in rural settings. ${ }^{7}$ The simplicity of RDT as a point of care test allows its mass deployment at all levels of malaria surveillance. ${ }^{8}$

As India targets malaria elimination, it is important to address potential foreseeable roadblocks. Malaria in India is heterogenous and unevenly distributed causing substantial morbidity in pregnant women and forest dwellers. ${ }^{9,10}$ Further, approximately $8 \%$ of the country's so-called tribal population shoulders approximately $46 \%$ of total malaria cases and $47 \%$ of deaths. ${ }^{11}$ Currently, the healthcare workers deployed in the public health system are supplemented by female village residents and volunteers who are trained to perform RDTs on suspected cases in the community. Their effectiveness is reduced in tribal regions because tribal people are significantly dependent on traditional healers and/or untrained or unqualified individuals, a practice that often delays accurate diagnosis and can lead to improper treatment. In addition to the efforts of the national control program to cover the entire nation especially focusing on the interior and remote areas of the country, it is important to explore new avenues that can expand access to RDTs to further enhance malaria surveillance ${ }^{12}$ and consequently improve timely treatment delivery.

The Indian private health sector provides general health services for a large proportion of the population. It includes 1) the formal sector (i.e., qualified practitioners, hospitals, nursing homes, and clinics) and 2) the informal sector (i.e., unqualified and unlicensed healthcare practitioners). For febrile individuals, the long queues, extended travel times, loss of wages, stock outs, and limited clinic operating hours either independently or in combination lead to dissatisfaction with the public health sector. Thus, they seek care in the private health sector. Government-licensed pharmacies often act as point of healthcare in resource-constrained settings owing to their accessibility, affordability (no consultation fee), ease of transaction, immediate treatment, and a general orientation toward consumer needs. According to household survey data, approximately half of febrile patients tend to approach private healthcare in rural areas of malaria endemic states. ${ }^{3}$ This situation is not unique to India. With respect to a large share of private-market drug sales, most countries in 
sub-Saharan Africa and Southeast Asia have already considered providing malaria RDTs to these outlets to enhance access to rapid diagnostics. ${ }^{13,14}$ Performance of private medicine retail outlets in sub-Sahara African countries was assessed in a review, and it was found that RDT uptake varied widely from 8 to $100 \%$, and disposal of medical waste and appropriate referrals to government health centers were identified as challenges. ${ }^{15}$

ACTwatch was launched in sub-Sahara African and in Southeast Asia in 2008 by Population Services International and has evaluated the deployment of both antimalarials and diagnostics through the private retail market. As part of ACTwatch, outlets that sell malaria diagnostics and drugs were surveyed in the four Greater Mekong Subregion countries of Lao PDR, Thailand, Myanmar, and Cambodia in 2015 and 2016. They sought information on compliance to standard malaria guidelines for diagnostics and disease management. The results revealed that stocking of malaria RDTs in the private sector was 58\% in Myanmar, 78\% in Lao PDR, $87 \%$ in Cambodia, and $91 \%$ in Thailand. All private facilities that stocked RDTs in Thailand and 94\% in Lao PDR were found to have quality-assured RDTs in stock. The majority (three out of four private facilities stocking RDTs) of pharmacies possessed quality-assured RDTs in Cambodia and Myanmar. However, non-certified RDTs were also available in $25 \%$ of facilities in Cambodia and in 17\% of facilities in Myanmar. Availability of first-line anti-malarial drugs for uncomplicated Plasmodium falciparum or Plasmodium vivax malaria was $70 \%$ in Cambodia and $40 \%$ in Lao PDR. In Myanmar the availability of first-line drugs was $42 \%$ for $P$. falciparum antimalarials and $56 \%$ for $P$. vivax, whereas in Thailand the availability was $19 \%$ for $P$. falciparum and $73 \%$ for $P$. vivax. ${ }^{16}$

In India, social marketing has been used to promote the use of condoms, urine pregnancy tests, and female contraception with substantial success. The Indian population is increasingly using home medical devices like pulse oximeters, glucometers, and sphygmomanometers. Hence, these empowerments can include RDT-based self-detection of malaria. Here we proposethat RDT availability be expanded to pharmacies and drug stores across India. As it is, the public health system is inadequate to ensure universal access to quality-assured RDTs, and the system could benefit from a complimentary contribution from the private sector. The ubiquitous presence of drug stores (mandatorily licensed under Indian law) has the potential to be an asset in the timely diagnosis of malaria. In addition to drug stores, local practitioners (formal and informal) can be suitably sensitized and trained to use RDTs and ACTs for malaria diagnosis and to appropriately treat as per the malaria species detected. They must also be primed and equipped with provisions to report positive cases to the public healthcare system. Feedback on usage of RDTs and data of malaria positives and treatments administered can be collated in the routine monthly meetings of the public healthcare system. This will ensure reporting of the numbers in the routine surveillance system and plan control measures. This will 1) prevent delays in diagnosis; 2) hasten treatment, which could substantially reduce morbidity, especially in young children; 3 ) be time and cost effective (average cost of subsidized malaria RDT is US\$0.4-0.5) for the patient to reduce direct and indirect costs; 4) reduce exposure to unqualified or untrained healthcare practitioners; and 5) help those who are malaria negative to seek timely healthcare for other ailments.
The challenges and risks of this approach are 1) incorrectly performed tests that give misleading results and lead to inappropriate treatments, which can have an adverse impact on the overall malaria control efforts; 2) the potential surge in sales of antimalarials and RDTs for personal and/or corporate profiteering; 3) the risk of nonprescription ACT procurement or nonreferral of RDT-positive patients as observed from a study collating information from five countries under ACT Consortium; ${ }^{17}$ 4) the need for training and supervision of lay staff in pharmacies and drug stores, especially of bivalent RDTs needed for detection of vivax malaria; 5) disposal of sharps/ medical waste; 6) reporting of results to public health authorities; 7) timely referral of RDT negative patients; and 8) poorquality RDTs compromising the true results.

The above impediments may be addressed via 1) training and sensitization of shop personnel along the lines of trainings done in public health system; 2) monitoring by supervisory public health system staff; 3 ) training the communities via simple-to-understand pictorial materials, workshops, video tutorials, and literature; 4) auditing mechanisms for vigilance on sales of RDTs and for records of positive patients and referrals; 5) anti-malarial drugs such as artemisinin combinations are covered under Schedule $\mathrm{H}$ of Drugs and Cosmetics Rules (2nd Amendment, 2006, Ministry of Health and Family Welfare) and hence cannot be sold without prescription, thereby preventing their abuse; 6) awareness campaigns to increase demand creation among communities because this will expand the market; 7) augmenting commodity supply systems and establishing incentive mechanisms for providers to enhance the uptake of RDTs; 8) ensuring medical waste disposal policy and adherence to it as followed currently for other diagnostics; and 9) encouraging drug stores and shop personnel to provide data through mobile applications or messaging services that are linked to routine surveillance systems in a centralized dashboard, as has been proposed recently. ${ }^{18}$ The digitized dashboard can be co-opted for other two eliminable vector-borne diseases viz lymphatic filariasis and visceral leishmaniasis. ${ }^{19}$

Despite the significant progress toward malaria elimination, India's path remains riddled with several obstacles. One such roadblock is universal access to malaria diagnostics. Hence, enhancing access to RDTs could be an empowerment tool for fast tracking the diagnosis and treatment of malaria in India.

Received February 22, 2021. Accepted for publication April 8, 2021.

Published online June 28, 2021.

Acknowledgments: We thank the Department of Science and Technology for the JC Bose fellowship to AS. The American Society of Tropical Medicine and Hygiene (ASTMH) assisted with publication expenses.

Author's addresses: Manju Rahi, Indian Council of Medical Research, New Delhi, India, E-mail: drmanjurahi@gmail.com. Amit Sharma, ICMR, National Institute of Malaria Research, Dwarka, New Delhi, India, and International Centre for Genetic Engineering and Biotechnology, New Delhi, India, E-mail: directornimr@gmail.com.

\section{REFERENCES}

1. WHO, 2020. World Malaria Report 2020. Geneva, Switzerland: World Health Organization. Available at: https://www.who.int/ publications/i/item/9789240015791. Accessed January 5, 2021.

2. National Vector Borne Disease Control Programme, 2016. Malaria Situation in India from 2016. Available at: https://pubmed.ncbi. nlm.nih.gov/24718394/. Accessed January 5, 2021. 
3. Anvikar AR et al., 2014. Antimalarial drug policy in India: past, present and future. Indian J Med Res 139: 205-215.

4. WHO, 2010. Guidelines for the Treatment of Malaria, 2nd edition. Geneva, Switzerland: World Health Organization. Available at: https://www.ncbi.nlm.nih.gov/books/NBK254223/. Accessed January 5, 2021.

5. Ministry of Health and Family Welfare, 2013. National Drug Policy on Malaria. Available at: https://nvbdcp.gov.in/WriteReadData/ 1892s/National-Drug-Policy-2013.pdf. Accessed January 5, 2021.

6. Rahi M, Ahmad SS, Sharma A, 2021. Coverage enhancement and community empowerment via commercial availability of the long-lasting nets for malaria in India. Pub Health Prac 2: 100133. doi: 10.1016/j.puhip.2021.100133.

7. Boyce MR, O'Meara WP, 2017. Use of malaria RDTs in various health contexts across sub-Saharan Africa: a systematic review. BMC Public Health 17: 470.

8. Ochodo E, Garner P, David Sinclair D, 2016. Achieving universal testing for malaria. BMJ 352: i107.

9. Pandey M, Rahi M and Sharma A, 2021. The Indian burden of malaria in pregnancy needs assessment. Med 2: 456-504. doi: 10.1016/j.medj.2021.04.017.

10. Ranjha R, Sharma A, 2021. Forest malaria: the prevailing obstacle for malaria control and elimination in India. BMJ Global Health 6: e005391. doi: 10.1136/ bmjgh-2021-00539.

11. Nema S, Ghanghoria P, Bharti PK, 2020. Malaria elimination in India: bridging the gap between control and elimination. Int Pediatr 57: 613-617.
12. Rahi $M$, Das $P$ and Sharma $A, 2021$. Malaria elimination in India requires additional surveillance mechanisms. J Pub Health fdab106. doi: 10.1093/pubmed/fdab106.

13. Maloney $\mathrm{K}$ et al., 2017. Expanding access to parasite-based malaria diagnosis through retail drug shops in Tanzania: evidence from a randomized trial and implications for treatment. Malaria 16: 6.

14. Mbonye AK et al., 2015. A cluster randomised trial introducing rapid diagnostic tests into registered drug shops in Uganda: impact on appropriate treatment of Malaria. PLoS One 10: e0129545.

15. Visser T et al., 2017. Introducing malaria rapid diagnostic tests in private medicine retail outlets: A systematic literature review. PLoS One 12: e0173093.

16. ACTwatch Group, Phok S, Phanalasy S, Thein ST, Likhitsup A, 2017. Private sector opportunities and threats to achieving malaria elimination in the Greater Mekong Subregion: results from malaria outlet surveys in Cambodia, the Lao PDR, Myanmar, and Thailand. Malar J 16: 180.

17. O'Boyle $S$ et al., 2020. Patients with positive malaria tests not given artemisinin-based combination therapies: a research synthesis describing under-prescription of antimalarial medicines in Africa. BMC Med 18: 17.

18. Rahi M, Sharma A, 2020. For malaria elimination India needs a platform for data integration. BMJ Glob Health 5: e004198.

19. Rahi M, Chaturvedi R, Das P and Sharma A, 2021. India can consider integration of three eliminable disease control programmes on malaria, lymphatic filariasis and visceral leishmaniasis. PLoS Pathogens. doi: 10.1371/journal.ppat.1009492. 